\title{
CONSERVATIVE FINITE VOLUME ELEMENT SCHEMES FOR THE COMPLEX MODIFIED KORTEWEG-DE VRIES EQUATION
}

\author{
JIN-LIANG YAN $^{a, *}$, LIANG-HONG ZHENG ${ }^{b}$ \\ ${ }^{a}$ Department of Mathematics and Computing \\ Wuyi University, Fujian, 354300, China \\ e-mail: yanjinliang3333@163.com \\ ${ }^{b}$ Department of Information and Technology \\ Nanping No. 1 Middle School, Fujian, 353000, China \\ e-mail: 413845939 @qq. com
}

\begin{abstract}
The aim of this paper is to build and validate a class of energy-preserving schemes for simulating a complex modified Korteweg-de Vries equation. The method is based on a combination of a discrete variational derivative method in time and finite volume element approximation in space. The resulting scheme is accurate, robust and energy-preserving. In addition, for comparison, we also develop a momentum-preserving finite volume element scheme and an implicit midpoint finite volume element scheme. Finally, a complete numerical study is developed to investigate the accuracy, conservation properties and long time behaviors of the energy-preserving scheme, in comparison with the momentum-preserving scheme and the implicit midpoint scheme, for the complex modified Korteweg-de Vries equation.
\end{abstract}

Keywords: mass, energy, momentum, finite volume element method, complex modified Korteweg-de Vries equation.

\section{Introduction}

The goal of this paper is to derive an energy-preserving finite volume element scheme for the nonlinear complex modified Korteweg-de Vries (CMKDV) equation. More specifically, we consider the following nonlinear equation:

$$
\begin{aligned}
& \frac{\partial u}{\partial t}+\frac{\partial^{3} u}{\partial x^{3}}+\alpha \frac{\partial\left(|u|^{2} u\right)}{\partial x}=0 \\
&-\infty<x<\infty, \quad t>0
\end{aligned}
$$

where $u(x, t)$ is a complex-valued function and $\alpha$ is a real constant. CMKDV is an important mathematical model, which is used to describe the nonlinear evolution of plasma waves (Karney et al., 1979), the propagation of transverse waves in a molecular chain model (Gorbacheva and Ostrovsky, 1983) and in a generalized elastic solid (Erbay and Suhubi, 1989; Erbay, 1998). i.e.,

If we decompose $u$ into its real and imaginary parts,

$$
u=p+i v \quad\left(i^{2}=-1\right)
$$

\footnotetext{
*Corresponding author
}

we obtain the following coupled modified Korteweg-de Vries (MKDV) equations:

$$
\begin{aligned}
& p_{t}+p_{x x x}+\alpha\left[\left(p^{2}+q^{2}\right) p\right]_{x}=0, \\
& q_{t}+q_{x x x}+\alpha\left[\left(p^{2}+q^{2}\right) q\right]_{x}=0 .
\end{aligned}
$$

The above coupled nonlinear equations describe the interaction of two orthogonally polarized transverse waves. In other words, $p$ and $q$ denote $y$-polarized and $z$-polarized transverse waves, respectively, propagating in the $x$-direction in an $x y z$ Cartesian coordinate system. The polarization angle $\theta$ is defined by $\tan \theta=q / p$. In some special case, such as $q=0$ or $p=0$ or $\theta=\theta_{0}$, which respectively correspond to the $0, \pi / 2$ and $\theta_{0}$ polarizations, the above coupled nonlinear equations reduce to a single MKDV equation. In this case, the CMKDV equation has the following analytical solution (Muslu and Erabay, 2003):

$$
u(x, t)=\sqrt{\frac{2 c}{\alpha}} \operatorname{sech}\left[\sqrt{c}\left(x-c t-x_{0}\right)\right] \exp \left(i \theta_{0}\right),
$$

which represents a solitary wave located around the position $x=x_{0}$ at time $t=0$, moving to the right with 
velocity $c$ and satisfying the boundary conditions $u \rightarrow 0$ as $x \rightarrow \pm \infty$.

We now assume that $u$ and all its derivatives converge to zero rapidly enough as $x \rightarrow \pm \infty$. Under these boundary conditions, there are some quantities which are of primary importance for Eqn. (1) (Karney et al., 1979): the conserved quantities of the solution,

$$
\mathcal{M}=\int_{-\infty}^{\infty} u \mathrm{~d} x, \quad \mathcal{K}=\int_{-\infty}^{\infty}|u|^{2} \mathrm{~d} x,
$$

also respectively called the mass, the momentum, and the energy

$$
\mathcal{H}=\int_{-\infty}^{\infty}\left(\frac{\alpha}{2}|u|^{4}-\left|\frac{\partial u}{\partial x}\right|^{2}\right) \mathrm{d} x .
$$

The ability of a numerical scheme to reproduce these quantities is extremely important, most particularly when they are conserved.

Deriving accurate and efficient numerical schemes which are well-adapted to describe the conservative properties of the CMKDV equation is a meaningful computational problem. For the numerical solution of the CMKDV equation, several methods have been developed. Muslu and Erabay (2003) proposed three different split-step Fourier schemes for the the CMKDV equation. Ismail $(2008 ; 2009)$ derived a Petrov-Galerkin method and a collocation method. Uddin et al. (2009) proposed a mesh-free collocation method, while Korkmaz and Dağ (2009) used a differential quadrature method to solve the CMKDV equation. Cai and Miao (2012) proposed an explicit multisymplectic Fourier scheme for the CMKDV equation.

The discrete variational derivative method (DVDM) is a class of structure-preserving methods, proposed by Furihata and Matsuo (2010), and it can retain the conservation/dissipation properties of the original equations. Up to now, the DVDM has been applied to many conservative or dissipative partial differential equations (PDEs). For example, Furihata and Mori (1996) designed a stable dissipative difference scheme for the Cahn-Hilliard equation. Koide and Furihata (2009) proposed four conservative difference schemes for the regularized long wave equation. Further, Matsuo and Furihata (2001) extended the general studies to complex-valued PDEs, while Yaguchi et al. (2010) extended the method to nonuniform grids. Matsuo and Kuramae (2012) proposed an alternating DVDM. Recently, Miyatake and Matsuo (2014) presented a general framework for constructing energy dissipative or conservative Galerkin schemes for time-dependent PDEs.

The finite volume element method (FVEM), as a type of important numerical tool for solving differential equations, has a long history. This method is also known as a box method in early references (Bank and Rose, 1987; Hackbusch, 1989; Costa et al., 2015), or as a generalized difference method (Li et al., 2000) in China. Cai (1991) and Ewing et al. (2000) presented the general error estimation framework for the FVEM for solving elliptic boundary value problems. The method has been widely used in several engineering fields, such as fluid mechanics, heat and mass transfer or petroleum engineering. Perhaps the most important property of the FVEM is that it can preserve the conservation laws (mass, momentum and heat flux) on each computational cell. This important property, combined with adequate accuracy and ease of implementation, has encouraged more people to do research in this field. It is worth noting that Zhang and $\mathrm{Lu}$ (2012) proposed a quadratic finite volume element scheme for the improved Boussinesq equation. Yan et al. (2016) reported a two-grid finite volume element scheme for nonlinear Sobolev equations. Wang et al. (2014) proposed an energy-preserving finite volume element scheme for the improved Boussinesq equation. In this paper, based on the DVDM and the FVEM, we develop an energy-preserving scheme which is accurate, unconditionally stable (with a long time computation ability) to solve the CMKDV numerically. On the other hand, for comparison, we also propose a momentum-preserving finite volume element scheme and an implicit midpoint finite volume element scheme.

The paper is organized as follows. In Section 2, we present the framework of the FVEM and define some notation. In Section 3, we derive the numerical schemes and prove the conservation properties. In Section 4, we present various numerical simulations to validate the accuracy, conservation properties and long time behaviors of our numerical schemes. Finally, we end the paper by a conclusion in Section 5.

\section{Notation and preliminaries}

For the application of the numerical method, we truncate the infinite interval to a finite interval $[a, b]$. In order to determine the solution of (1), we assume that $u(x, t)$ satisfies the periodic boundary condition $u(a, t)=u(b, t)$ for $(x, t) \in[a, b] \times[0, T]$. In addition, we assume that the interval $[a, b]$ is sufficiently large, such that the solution (2) is a good approximation to the periodic solution for quite a large time interval.

Firstly, we discretize the domain $I=[a, b]$ into a grid $T_{h}$ with nodes

$$
a=x_{0}<x_{1}<x_{2}<\cdots<x_{n-1}<x_{n}=b,
$$

where $h=x_{i}-x_{i-1}$ denotes the length of the element $I_{i}$ and $h=(b-a) / N$.

Then we place a dual grid $T_{h}^{*}$ with nodes

$$
a=x_{0}<x_{1 / 2}<x_{3 / 2}<\cdots<x_{n-1 / 2}<x_{n}=b,
$$

where $x_{i-1 / 2}=\left(x_{i-1}+x_{i}\right) / 2, I_{i}^{*}=\left[x_{i-1 / 2}, x_{i+1 / 2}\right]$, 
$i=1,2, \ldots, N . I_{0}^{*}=\left[x_{0}, x_{1 / 2}\right],(i=1,2, \ldots, N-1)$, and $I_{N}^{*}=\left[x_{N-1 / 2}, x_{N}\right]$ denote the dual elements.

The trial space $U_{h}$ is taken as the linear element space with respect to $T_{h}$. The basis function with respect to $x_{i}$ is

$$
\phi_{i}(x)= \begin{cases}1-h^{-1}\left|x-x_{i}\right|, & x_{i-1} \leq x \leq x_{i+1}, \\ 0, & \text { elsewhere }\end{cases}
$$

and any $u_{h} \in U_{h}$ has the following expression:

$$
u_{h}=\sum_{i=1}^{N} u_{i} \phi_{i}(x)
$$

where $u_{i}=u_{h}\left(x_{i}, t\right)$. On the element $I_{i}$ we have

$$
\begin{gathered}
u_{h}=u_{i-1}(1-\xi)+u_{i} \xi, \\
u_{h}^{\prime}=\frac{1}{h}\left(u_{i}-u_{i-1}\right), \quad x \in I_{i},
\end{gathered}
$$

where $i=1,2, \ldots, N$ and $\xi=\left(x-x_{i-1}\right) / h$.

Analogously, the test function space $V_{h}$ is chosen as a space of piecewise constant functions. The basis function at $x_{i}$ is

$$
\psi_{j}(x)= \begin{cases}1, & x_{j-1 / 2} \leq x \leq x_{j+1 / 2}, \\ 0, & \text { elsewhere }\end{cases}
$$

and any $v_{h} \in V_{h}$ has the form

$$
v_{h}=\sum_{i=1}^{N} v_{i} \psi_{i}(x)
$$

where $v_{i}=v_{h}\left(x_{i}, t\right)$.

The numerical solution is denoted by $U_{k}^{m}=$ $u(k \Delta x, m \Delta t)$, where $\Delta x$ is the space mesh size and $\Delta t$ is the time mesh size. Here we adopt a uniform time discretization $t^{0}, t^{1}, \ldots$, with a constant time step $\Delta t>0$.

\section{Numerical schemes}

In this section, we develop the proposed schemes for the CMKDV equation and prove its conservation properties.

3.1. Derivation of the proposed schemes. In order to derive the proposed schemes, we define the "local energy" of the CMKDV equation (1) as

$$
G\left(u, u_{x}\right)=\frac{\alpha}{2}|u|^{4}-\left|\frac{\partial u}{\partial x}\right|^{2} .
$$

Thus Eqn. (1) can be written as

$$
u_{t}=-\frac{\partial}{\partial x}\left(\frac{\delta G}{\delta \bar{u}}\right)
$$

where $\delta G / \delta \bar{u}$ is the variational derivative of $G\left(u, u_{x}\right)$ defined by

$$
\frac{\delta G}{\delta \bar{u}}=\frac{\partial G}{\partial \bar{u}}-\frac{\partial}{\partial x}\left(\frac{\partial G}{\partial \overline{u_{x}}}\right) .
$$

In order to derive the energy-preserving scheme, we first define a scheme of the local energy as

$$
G_{d, k}(U)=\frac{\alpha}{2}\left|U_{k}\right|^{4}-\frac{\left|\delta_{k}^{+} U_{k}\right|^{2}+\left|\delta_{k}^{-} U_{k}\right|^{2}}{2},
$$

where $\delta_{k}^{+} U_{k}=\left(U_{k+1}-U_{k}\right) / h, \delta_{k}^{-} U_{k}=\left(U_{k}-U_{k-1}\right) / h$.

Then we can obtain a scheme of the variational derivative

$$
\begin{aligned}
& \frac{\delta G_{d}}{\delta\left(\bar{U}^{(m+1)}, \bar{U}^{(m)}\right)_{k}} \\
& =\frac{\alpha}{2}\left(\left|U_{k}^{(m+1)}\right|^{2}+\left|U_{k}^{(m)}\right|^{2}\right) U_{k}^{\left(m+\frac{1}{2}\right)} \\
& \quad+\delta_{k}^{\langle 2\rangle} U_{k}^{\left(m+\frac{1}{2}\right)},
\end{aligned}
$$

where $\delta_{k}^{\langle 2\rangle}$ is the standard central difference operator for $\partial^{2} / \partial x^{2}, \quad$ and $U_{k}^{\left(m+\frac{1}{2}\right)}=\left(U_{k}^{(m+1)}+U_{k}^{(m)}\right) / 2$.

The scheme (5) is obtained by the following difference (Furihata and Matsuo, 2010):

$$
\begin{aligned}
\sum_{k=0}^{N} \prime \prime & \left(G_{d, k}\left(U^{(m+1)}\right)-G_{d, k}\left(U^{(m)}\right)\right) \Delta x \\
= & \sum_{k=0}^{N} " \prime\left\{\frac{\delta G_{d}}{\delta\left(U^{(m+1)}, U^{(m)}\right)_{k}}\left(U_{k}^{(m+1)}-U_{k}^{(m)}\right)\right. \\
& \left.+\frac{\delta G_{d}}{\delta\left(\bar{U}^{(m+1)}, \bar{U}^{(m)}\right)_{k}}\left(\bar{U}_{k}^{(m+1)}-\bar{U}_{k}^{(m)}\right)\right\} \Delta x \\
& +(\text { boundary terms) }
\end{aligned}
$$

where

$$
\sum_{k=0}^{N}{ }^{\prime \prime} f_{k} \triangleq \frac{1}{2} f_{0}+f_{1}+\cdots+f_{N-1}+\frac{1}{2} f_{N}
$$

is the trapezoidal rule and the boundary terms vanish due to the periodic boundary condition.

On the other hand, for comparison, we derive another scheme of the variational derivative corresponding to the momentum-preserving scheme:

$$
\begin{aligned}
& \frac{\delta G_{d}}{\delta\left(\bar{U}_{+}^{\left(m+\frac{1}{2}\right)}, \bar{U}_{-}^{\left(m+\frac{1}{2}\right)}\right)_{k}} \\
& =\frac{\alpha}{4}\left(\left|\left(U_{+}^{\left(m+\frac{1}{2}\right)}\right)_{k}\right|^{2}+\left|\left(U_{-}^{\left(m+\frac{1}{2}\right)}\right)_{k}\right|^{2}\right) \\
& \quad \times\left(\left(U_{+}^{\left(m+\frac{1}{2}\right)}\right)_{k}+\left(U_{-}^{\left(m+\frac{1}{2}\right)}\right)_{k}\right) \\
& \quad+\frac{1}{2} \delta_{k}^{\langle 2\rangle}\left(\left(U_{+}^{\left(m+\frac{1}{2}\right)}\right)_{k}+\left(U_{-}^{\left(m+\frac{1}{2}\right)}\right)_{k}\right),
\end{aligned}
$$


where

$$
\begin{aligned}
& \left(U_{+}^{\left(m+\frac{1}{2}\right)}\right)_{k}=\frac{1}{2}\left(U_{k+1}^{(m+1)}+U_{k+1}^{(m)}\right), \\
& \left(U_{-}^{\left(m+\frac{1}{2}\right)}\right)_{k}=\frac{1}{2}\left(U_{k-1}^{(m+1)}+U_{k-1}^{(m)}\right) .
\end{aligned}
$$

From the previous discretization of the discrete variational derivative given by Eqns. (5) and (6), we introduce the following energy-preserving and momentum-preserving finite volume element schemes:

$$
\begin{gathered}
\left(\delta_{m}^{+} U^{(m)}, \psi_{i}\right)=-\left(\frac{\partial}{\partial x}\left(\frac{\delta G_{d}}{\delta\left(\bar{U}^{(m+1)}, \bar{U}^{(m)}\right)}\right), \psi_{i}\right), \\
\left(\delta_{m}^{+} U^{(m)}, \psi_{j}\right)=-\left(\frac{\partial}{\partial x}\left(\frac{\delta G_{d}}{\delta\left(\bar{U}_{+}^{\left(m+\frac{1}{2}\right)}, \bar{U}_{-}^{\left(m+\frac{1}{2}\right)}\right)}\right), \psi_{j}\right),
\end{gathered}
$$

where we set $\delta_{m}^{+} U^{(m)}=\left(U^{(m+1)}-U^{(m)}\right) / \Delta t, U^{m} \in$ $U_{h}, \psi_{i}, \psi_{j} \in V_{h}, i, j=1,2, \ldots, N$, and $m=$ $0,1, \ldots, T / \Delta t$.

Finally, for comparison, we also present an implicit midpoint finite volume element scheme,

$$
\begin{aligned}
& \left(\delta_{m}^{+} U^{(m)}, \psi_{k}\right) \\
& \quad=-\left(\left(\alpha\left|U^{\left(m+\frac{1}{2}\right)}\right|^{2} U^{\left(m+\frac{1}{2}\right)}+U_{x x}^{\left(m+\frac{1}{2}\right)}\right)_{x}, \psi_{k}\right),
\end{aligned}
$$

where $U^{m} \in U_{h}, \psi_{k} \in V_{h}, k=1,2, \ldots, N$.

\subsection{Conservation properties of the schemes.}

Theorem 1. (Mass conservation law) Let $U=U^{(m)}$ be the solution of (7), and assume that the boundary conditions satisfy

$$
\left[-\frac{\delta G_{d}}{\delta\left(\bar{U}^{(m+1)}, \bar{U}^{(m)}\right)}\right]_{x=a}^{b}=0 .
$$

Then the solution of the scheme (7) satisfies

$$
\int_{a}^{b} U^{(m)} \mathrm{d} x=\text { const. }
$$

Proof. We have

$$
\begin{aligned}
\frac{1}{\Delta t} & \int_{a}^{b}\left(U^{(m+1)}-U^{(m)}\right) \mathrm{d} x \\
& =\int_{a}^{b}\left(\frac{U^{(m+1)}-U^{(m)}}{\Delta t}\right) \mathrm{d} x \\
& =-\int_{a}^{b} \frac{\partial}{\partial x}\left(\frac{\delta G_{d}}{\delta\left(\bar{U}^{(m+1)}, \bar{U}^{(m)}\right)}\right) \mathrm{d} x \\
& =\left[-\frac{\delta G_{d}}{\delta\left(\bar{U}^{(m+1)}, \bar{U}^{(m)}\right)}\right]_{x=a}^{b}=0 .
\end{aligned}
$$

Theorem 2. (Energy conservation law) Let $U=U^{(m)}$ be the solution of (7), and assume that the boundary conditions satisfy

$$
\left[-\left|\frac{\delta G_{d}}{\delta\left(U^{(m+1)}, U^{(m)}\right)}\right|^{2}\right]_{x=a}^{b}=0 .
$$

Then the solution of the scheme (7) satisfies

$$
\int_{a}^{b} G_{d}\left(U^{(m)}(x)\right) \mathrm{d} x=\text { const. }
$$

Proof. We have

$$
\begin{aligned}
& \frac{1}{\Delta t} \int_{a}^{b}\left(G_{d}\left(U^{(m+1)}\right)-G_{d}\left(U^{(m)}\right)\right) \mathrm{d} x \\
&=\int_{a}^{b}\left\{\frac{\delta G_{d}}{\delta\left(U^{(m+1)}, U^{(m)}\right)} \frac{U^{(m+1)}-U^{(m)}}{\Delta t}\right. \\
&\left.+\frac{\delta G_{d}}{\delta\left(\bar{U}^{(m+1)}, \bar{U}^{(m)}\right)} \frac{\bar{U}^{(m+1)}-\bar{U}^{(m)}}{\Delta t}\right\} \mathrm{d} x \\
&=-\int_{a}^{b}\left\{\frac{\delta G_{d}}{\delta\left(U^{(m+1)}, U^{(m)}\right)} \frac{\partial}{\partial x}\left(\frac{\delta G_{d}}{\delta\left(\bar{U}^{(m+1)}, \bar{U}^{(m)}\right)}\right)\right. \\
&+\left.\frac{\delta G_{d}}{\delta\left(\bar{U}^{(m+1)}, \bar{U}^{(m)}\right)} \frac{\partial}{\partial x}\left(\frac{\delta G_{d}}{\delta\left(U^{(m+1)}, U^{(m)}\right)}\right)\right\} \mathrm{d} x \\
&=-\int_{a}^{b} \frac{\partial}{\partial x}\left(\frac{\delta G_{d}}{\delta\left(U^{(m+1)}, U^{(m)}\right)} \frac{\delta G_{d}}{\delta\left(\bar{U}^{(m+1)}, \bar{U}^{(m)}\right)}\right) \mathrm{d} x \\
&=-\int_{a}^{b} \frac{\partial}{\partial x}\left(\left|\frac{\delta G_{d}}{\delta\left(U^{(m+1)}, U^{(m)}\right)}\right|^{2}\right) \mathrm{d} x \\
&= {\left[-\left|\frac{\delta G_{d}}{\delta\left(U^{(m+1)}, U^{(m)}\right)}\right|^{b}\right]_{x=a}=0 . }
\end{aligned}
$$

Theorem 3. (Mass conservation law) Let $U=U^{(m)}$ be the solution of (8), and assume that the boundary conditions satisfy

$$
\left[-\frac{\delta G_{d}}{\delta\left(\bar{U}_{+}^{(m)}, \bar{U}_{-}^{(m)}\right)}\right]_{x=a}^{b}=0 .
$$

Then the solution of the scheme (8) satisfies

$$
\int_{a}^{b} U^{(m)} \mathrm{d} x=\text { const. }
$$

The proof is similar to that of Theorem 1

Theorem 4. (Momentum conservation law) Let $U=$ $U^{(m)}$ be the solution of (8), and assume that the boundary 
conditions satisfy

$$
\begin{aligned}
& {\left[-\bar{U}^{(m+1)} \frac{\delta G_{d}}{\delta\left(\bar{U}_{+}^{(m)}, \bar{U}_{-}^{(m)}\right)}\right]_{x=a}^{b}=0,} \\
& {\left[-U^{(m)} \frac{\delta G_{d}}{\delta\left(U_{+}^{(m)}, U_{-}^{(m)}\right)}\right]_{x=a}^{b},} \\
& {\left[G_{d}\left(\bar{U}^{(m+1)}\right)\right]_{x=a}^{b}=0, \quad\left[G_{d}\left(U^{(m)}\right)\right]_{x=a}^{b}=0 .}
\end{aligned}
$$

then the solution of scheme (8) satisfies

$$
\int_{a}^{b}\left|U^{(m)}\right|^{2} \mathrm{~d} x=\text { const }
$$

Proof. We have

$$
\begin{aligned}
& \frac{1}{\Delta t} \int_{a}^{b}\left[\left|U^{(m+1)}\right|^{2}-\left|U^{(m)}\right|^{2}\right] \mathrm{d} x \\
& =\frac{1}{\Delta t} \int_{a}^{b}\left[U^{(m+1)} \bar{U}^{(m+1)}-U^{(m)} \bar{U}^{(m)}\right] \mathrm{d} x \\
& =\int_{a}^{b}\left[\frac{U^{(m+1)}-U^{(m)}}{\Delta t} \bar{U}^{(m+1)}\right. \\
& \left.+\frac{\bar{U}^{(m+1)}-\bar{U}^{(m)}}{\Delta t} U^{(m)}\right] \mathrm{d} x \\
& =\int_{a}^{b}\left[\delta_{m}^{+} U^{(m)} \bar{U}^{(m+1)}+\delta_{m}^{+} \bar{U}^{(m)} U^{(m)}\right] \mathrm{d} x \\
& =-\int_{a}^{b}\left[\frac{\partial}{\partial x}\left(\frac{\delta G_{d}}{\delta\left(\bar{U}_{+}^{(m)}, \bar{U}_{-}^{(m)}\right)}\right) \bar{U}^{(m+1)}\right. \\
& \left.+\frac{\partial}{\partial x}\left(\frac{\delta G_{d}}{\delta\left(U_{+}^{(m)}, U_{-}^{(m)}\right)}\right) U^{(m)}\right] \mathrm{d} x \\
& =\int_{a}^{b} \frac{\partial \bar{U}^{(m+1)}}{\partial x} \frac{\delta G_{d}}{\delta\left(\bar{U}_{+}^{(m)}, \bar{U}_{-}^{(m)}\right)} \mathrm{d} x \\
& +\int_{a}^{b} \frac{\partial U^{(m)}}{\partial x} \frac{\delta G_{d}}{\delta\left(U_{+}^{(m)}, U_{-}^{(m)}\right)} \mathrm{d} x \\
& =\int_{a}^{b} \frac{\partial G_{d}\left(\bar{U}^{(m+1)}\right)}{\partial x} \mathrm{~d} x+\int_{a}^{b} \frac{\partial G_{d}\left(U^{(m)}\right)}{\partial x} \mathrm{~d} x \\
& =\left[G_{d}\left(\bar{U}^{(m+1)}\right)\right]_{x=a}^{b}+\left[G_{d}\left(U^{(m)}\right)\right]_{x=a}^{b}=0 .
\end{aligned}
$$

Theorem 5. (Mass conservation law) Let $U=U^{(m)}$ be the solution of (9). Then the discrete mass $M$ is constant,

$$
\int_{a}^{b} U^{(m)} \mathrm{d} x=\text { const. }
$$

The proof is also similar to that of Theorem 1

\section{Numerical experiments}

In this section, we shall present some numerical examples to validate the accuracy, conservation properties and long time behaviors of the proposed schemes. In the sequel, we will use $L_{\infty}=\max _{0 \leq i \leq N-1}\left|u\left(x_{i}, t_{n}\right)-U_{i}^{(n)}\right|$ and order $=\log _{2}\left(\left\|u^{n}-U_{2 h}^{(n)}\right\|_{\infty} /\left\|u^{(n)}-U_{h}^{(n)}\right\|_{\infty}\right)$ to evaluate the accuracy and the order of convergence of the proposed methods. On the other hand, the conserved quantities at the discrete level and the relative errors of the invariants corresponding to (3) and (4) on the $n$-th time level can be defined as

$$
\begin{aligned}
M\left(U^{(n)}\right)= & h \sum_{k=0}^{N-1} U_{k}^{(n)}, \\
K\left(U^{(n)}\right)= & h \sum_{k=0}^{N-1}\left|U_{k}^{(n)}\right|^{2}, \\
H\left(U^{(n)}\right)= & \frac{h}{2} \sum_{k=0}^{N-1}\left[\alpha\left|U_{k}^{(n)}\right|^{4}-\left(\left|\delta_{k}^{+} U_{k}^{(n)}\right|^{2}\right.\right. \\
& \left.\left.+\left|\delta_{k}^{-} U_{k}^{(n)}\right|^{2}\right)\right], \\
\text { relative error }= & \lg \left(\frac{\left|I^{(n)}-I^{(0)}\right|}{\left|I^{(0)}\right|}\right),
\end{aligned}
$$

where $I^{(n)}$ denotes the global quantities on the $n$-th time level.

4.1. Single solitary wave. In this example, we adopt $\alpha=2$ and choose the following initial condition:

$$
u(x, 0)=\sqrt{c} \operatorname{sech}\left[\sqrt{c}\left(x-x_{0}\right)\right] \exp (i \theta),
$$

where $c$ denotes the speed of the wave, while $\theta$ represents the phase.

We consider the following tests:

(i) We first consider the accuracy of the proposed schemes. Here we choose $x_{0}=-10 \mathrm{~m}, c=1.5 \mathrm{~m} / \mathrm{s}$, $\theta=\pi / 2$, and $-30 \leq x \leq 30$. Tables 1 and 2 present respectively the $L_{\infty}$ error and the order of convergence of the three schemes in space and time. It is very clear that the energy-preserving finite volume element method (EFVEM) produces more accurate solutions than the momentum-preserving finite volume element method (MFVEM) and the finite volume element method (FVEM). On the other hand, it is noted that the $L_{\infty}$ error is exactly the same for the EFVEM and the FVEM in Table 1. In fact, this is because the EFVEM and the FVEM adopt a similar FVEM discretization in space. In Fig. 1, we display the $L_{\infty}$ errors of the proposed schemes at time $T=8 \mathrm{~s}$ 
(ii) Then we consider the conservation properties and the long time behaviors of the proposed schemes. Here we choose $x_{0}=15 \mathrm{~m}, c=1 \mathrm{~m} / \mathrm{s}, \theta=\pi / 2$ and $0 \leq x \leq 30$. Figure 2 presents the numerical results of the energy-preserving scheme for $t$ in $[0,80]$. It is clearly seen that the energy-preserving scheme can precisely conserve the discrete mass and energy. Figure 3 presents the conservation properties of the FVEM and the MFVEM. Figure 4 presents the numerical solution at time $T=1000 \mathrm{~s}$ and the relative errors of the invariants for $t$ in $[0, T]$.

4.2. Interaction of two solitary waves. In this example, we first study the interaction between a $y$-polarized solitary wave $\left(\theta_{1}=0\right)$ and a $z$-polarized solitary wave $\left(\theta_{2}=\pi / 2\right)$. Here $\theta_{1}$ and $\theta_{2}$ denote the polarization angles of the waves. This example is from the work of Korkmaz and Dağ (2009). Here $\alpha=2$ and the initial condition is given by

$$
\begin{aligned}
u(x, 0)= & \sqrt{2} \operatorname{sech}(\sqrt{2}(x-25)) \\
& +i \frac{\sqrt{2}}{2} \operatorname{sech}\left(\frac{\sqrt{2}}{2}(x-48)\right) .
\end{aligned}
$$

This equation corresponds to two solitary waves, one initially located at $x_{1}=25 \mathrm{~m}$ and moving to the right with speed $2 \mathrm{~m} / \mathrm{s}$, the other initially located at $x_{2}=48 \mathrm{~m}$ and moving to the right with speed $0.5 \mathrm{~m} / \mathrm{s}$. The problem is solved on the interval $0 \leq x \leq 100$ and the computations are done up to time $T=30 \mathrm{~s}$. Figure 5 presents the modulus of the solution and the relative errors of the invariants for the EFVEM. Figure 6 displays the real and imaginary parts of the numerical solution. These results are obtained using a space step $h=0.2$ and a time step $\Delta t=0.1$. It is clearly seen that the taller wave is initially

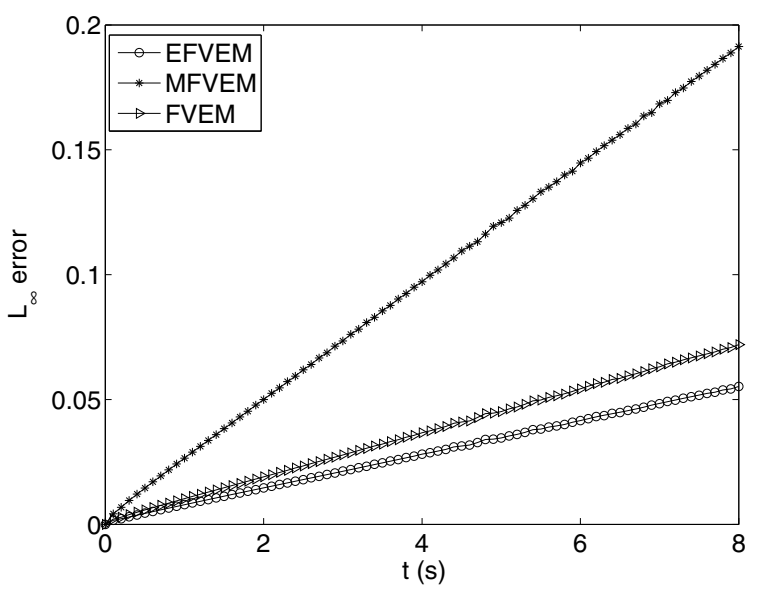

Fig. 1. $L_{\infty}$ error of the proposed schemes with $h=\Delta t=0.1$, $x_{0}=-10 \mathrm{~m}, c=1.5 \mathrm{~m} / \mathrm{s}, \theta=\pi / 2$, and $T=8 \mathrm{~s}$. located to the left of the shorter one. Then the taller one gradually catches up the shorter one and an interaction occurs, since the taller one is faster than the shorter one. After the interaction, the taller wave again moves away from the shorter one and continues to travel forward. In addition, we note that there is a small tail after the shorter wave, which is in agreement with the results of Ismail (2008). On the other hand, the energy-preserving scheme can also exactly conserve the mass and the energy at the discrete level. It is also noted that the relative error of the total momentum is bounded.

Finally, we also study the interaction of two $y$-polarized solitary waves $\left(\theta_{1}=\theta_{2}=0\right)$ like Muslu and Erabay (2003). The value of $\alpha$ is also chosen as $\alpha=2$, and the initial condition is given by

$$
\begin{aligned}
u(x, 0)= & \sqrt{2} \operatorname{sech}(\sqrt{2}(x-25)) \\
& +\frac{\sqrt{2}}{2} \operatorname{sech}\left(\frac{\sqrt{2}}{2}(x-48)\right) .
\end{aligned}
$$

The problem is also solved on the interval $0 \leq x \leq 100$ and the computation is also performed up to time $T=$ $30 \mathrm{~s}$. Here the results are also obtained using the space step $h=0.2$ and the time step the $\Delta t=0.1$. Figure 7 displays the modulus of the solution and the relative errors of the invariants for the EFVEM. Figure 8 presents the real and imaginary parts of the numerical solution. As can be seen from Fig. 7 between the taller and shorter waves there is an elastic interaction, and there is no small tail following the shorter one. This phenomenon again indicates that the interaction of two solitary waves is more stable when the two solitary waves are polarized in the same direction, which is in agreement with the findings of Muslu and Erabay (2003).

4.3. Wave break-up. In this example, we study the process of a solitary wave break up into two solitary waves. This example is from the work of Korkmaz and Dağ (2009). To this end, the initial condition is given by

$$
u(x, 0)=2 \operatorname{sech}(x) \exp \left(i \theta_{0}\right),
$$

where the polarization angle is chosen as $\theta_{0}=\pi / 4$. The problem is solved over the interval $-30 \leq x \leq$ 30 and the computation is performed up to time $T=$ $2.5 \mathrm{~s}$. Figure 9 presents the modulus of numerical solution and the relative errors of the invariants for the energy-preserving scheme. Figure 10 displays the real part and the imaginary part of the numerical solution. These results are obtained by using the space step $h=0.2$ and the time step $\Delta t=0.01$. As can be seen from Fig. 9 . initially the wave is a solitary one, but as time increases, it breaks up into two solitary waves and becomes thinner and higher compared with the initial state, which is in agreement with the results of Korkmaz and Dağ (2009). 
Table 1. Space $L_{\infty}$ errors of the proposed schemes with $N=1000, \Delta t=0.0001,-30 \leq x \leq 30$.

\begin{tabular}{|lllllll|}
\hline$h$ & EFVEM & order & MFVEM & order & FVEM & order \\
\hline \hline $1 / 2$ & $7.7000 \mathrm{e}-03$ & - & $1.5100 \mathrm{e}-02$ & - & $7.7000 \mathrm{e}-03$ & - \\
$1 / 4$ & $2.5000 \mathrm{e}-03$ & 1.6229 & $6.8000 \mathrm{e}-03$ & 1.1509 & $2.5000 \mathrm{e}-03$ & 1.6229 \\
$1 / 8$ & $6.3800 \mathrm{e}-04$ & 1.9703 & $1.5000 \mathrm{e}-03$ & 2.1806 & $6.3800 \mathrm{e}-04$ & 1.9703 \\
$1 / 16$ & $1.6152 \mathrm{e}-04$ & 1.9818 & $3.7071 \mathrm{e}-04$ & 2.0166 & $1.6152 \mathrm{e}-04$ & 1.9818 \\
$1 / 32$ & $4.0359 \mathrm{e}-05$ & 2.0008 & $9.1842 \mathrm{e}-05$ & 2.0131 & $4.0359 \mathrm{e}-05$ & 2.0008 \\
\hline
\end{tabular}

Table 2. Time $L_{\infty}$ errors of the proposed schemes with $h=1 / 32, T=1 s,-30 \leq x \leq 30$.

\begin{tabular}{|lllllll|}
\hline$\Delta t$ & EFVEM & order & MFVEM & order & FVEM & order \\
\hline \hline $1 / 2$ & $2.5000 \mathrm{e}-02$ & - & $3.3000 \mathrm{e}-02$ & - & $3.2600 \mathrm{e}-02$ & - \\
$1 / 4$ & $6.8000 \mathrm{e}-03$ & 1.8783 & $9.4000 \mathrm{e}-03$ & 1.8117 & $8.9000 \mathrm{e}-03$ & 1.8730 \\
$1 / 8$ & $1.7000 \mathrm{e}-03$ & 2.0000 & $2.8000 \mathrm{e}-03$ & 1.7472 & $2.3000 \mathrm{e}-03$ & 1.9522 \\
$1 / 16$ & $4.4875 \mathrm{e}-04$ & 1.9216 & $1.1000 \mathrm{e}-03$ & 1.3479 & $5.8345 \mathrm{e}-04$ & 1.9790 \\
$1 / 32$ & $1.2334 \mathrm{e}-04$ & 1.8633 & $6.7736 \mathrm{e}-04$ & 0.6995 & $1.5761 \mathrm{e}-04$ & 1.8882 \\
\hline
\end{tabular}

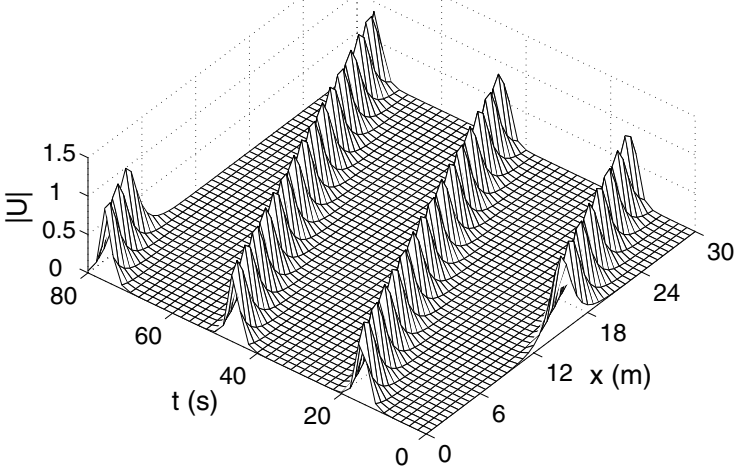

(a)

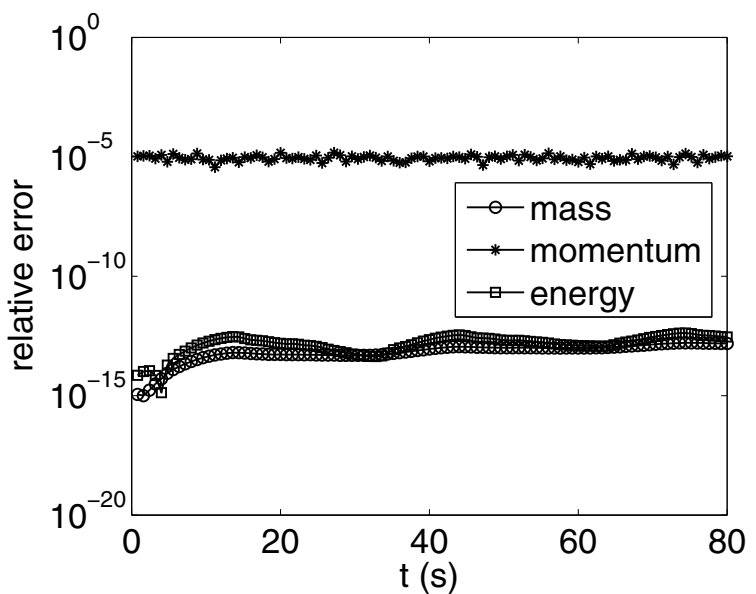

(b)

Fig. 2. Numerical results of the energy-preserving scheme: numerical solution (a), relative errors of invariants (b), when $h=0.2$, $\Delta t=0.1, x_{0}=15 \mathrm{~m}, c=1 \mathrm{~m} / \mathrm{s}, \theta=\pi / 2, T=80 \mathrm{~s}$, and $0 \leq x \leq 30$.

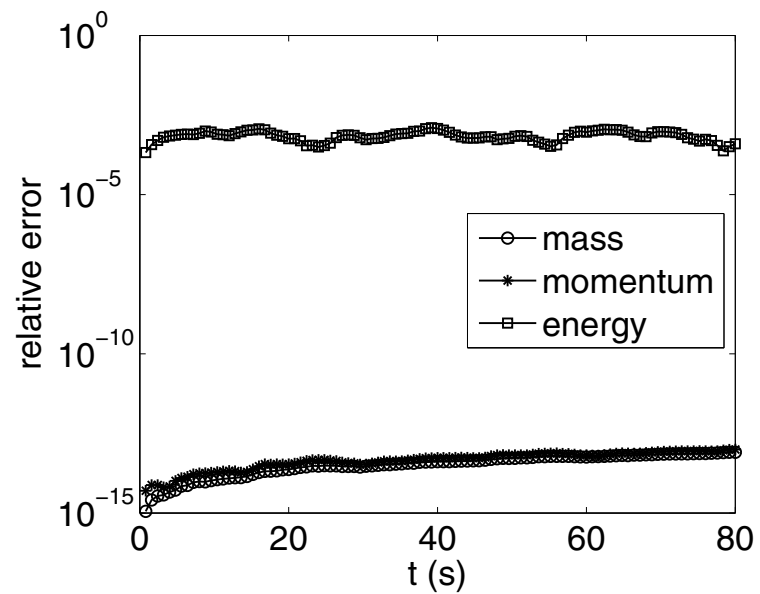

(a)

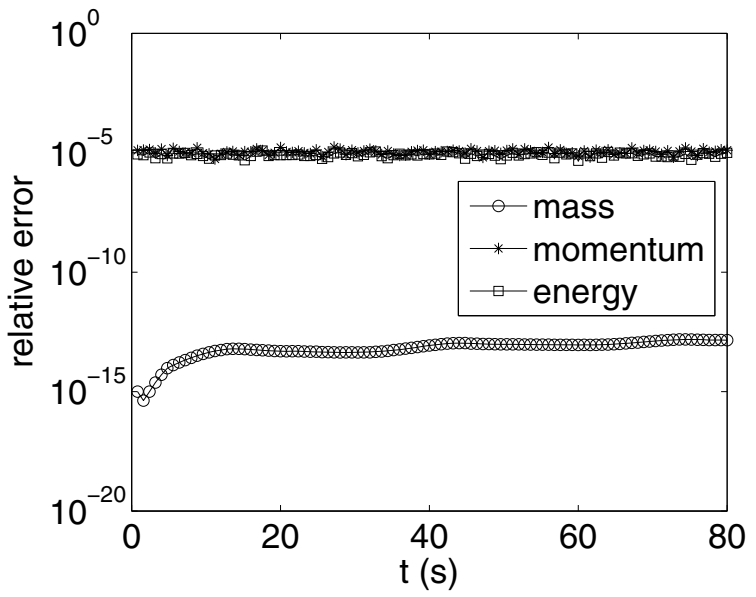

(b)

Fig. 3. Relative errors of the invariants: momentum-preserving scheme (a), finite volume element scheme (b), when $h=0.2, \Delta t=$ $0.1, x_{0}=15 \mathrm{~m}, c=1 \mathrm{~m} / \mathrm{s}, \theta=\pi / 2, T=80 \mathrm{~s}$, and $0 \leq x \leq 30$. 


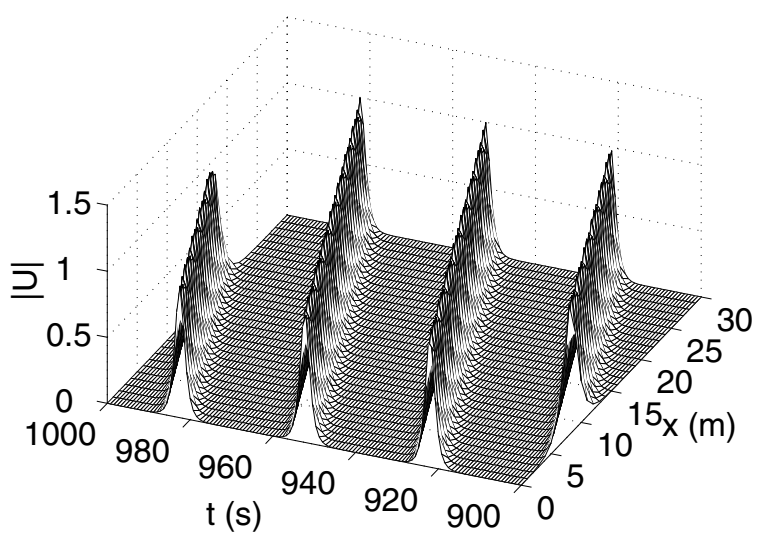

(a)

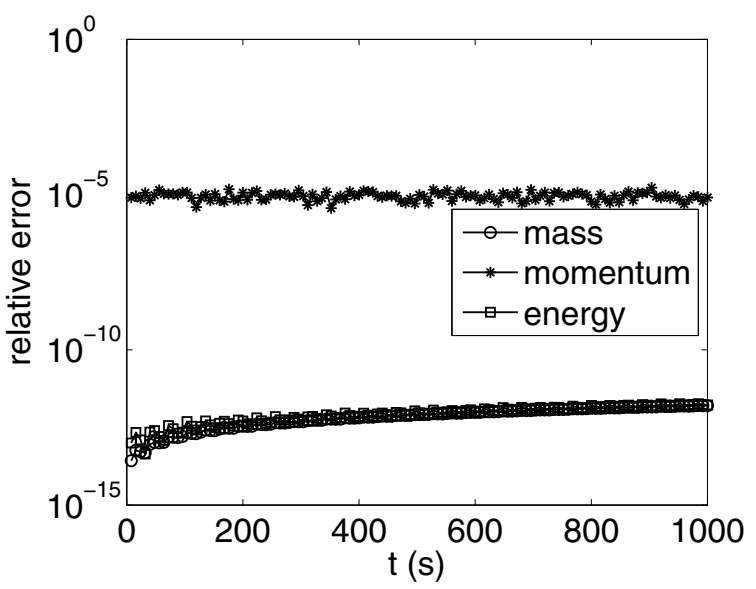

(b)

Fig. 4. Numerical results of the energy-preserving scheme: numerical solution (a), relative errors of invariants (b), when $h=0.2$, $\Delta t=0.1, x_{0}=15 \mathrm{~m}, c=1 \mathrm{~m} / \mathrm{s}, \theta=\pi / 2, T=1000 \mathrm{~s}$, and $0 \leq x \leq 30$.

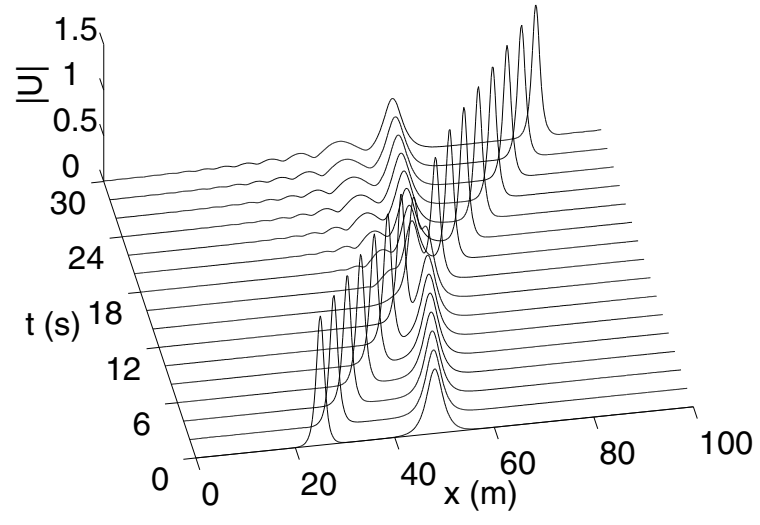

(a)

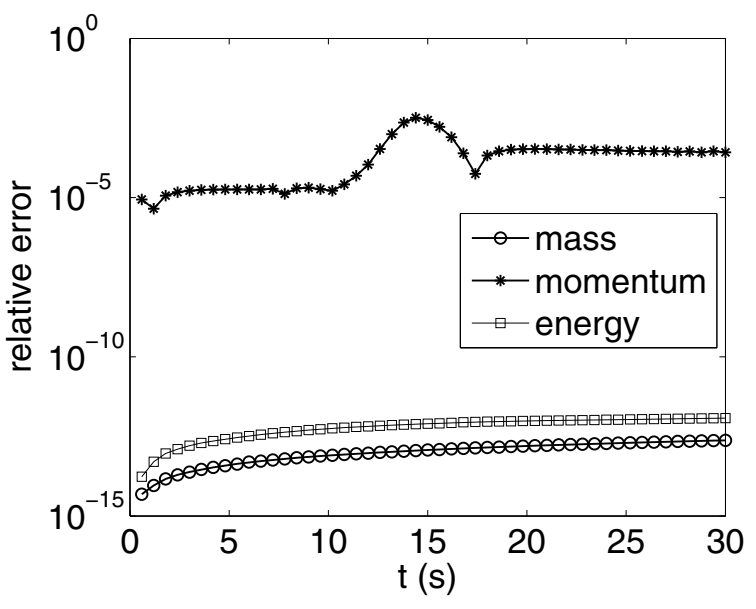

(b)

Fig. 5. Modulus (a) and relative errors of the invariants (b) of the numerical solution obtained for the interaction between $y$-polarized and $z$-polarized solitary waves using the energy-preserving scheme with $h=0.2, \Delta t=0.1, x_{1}=25 \mathrm{~m}, x_{2}=48 \mathrm{~m}, T=30 \mathrm{~s}$, and $0 \leq x \leq 100$.

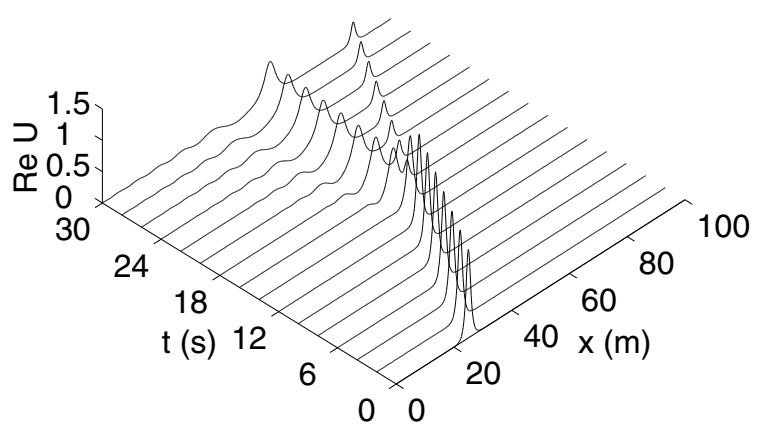

(a)

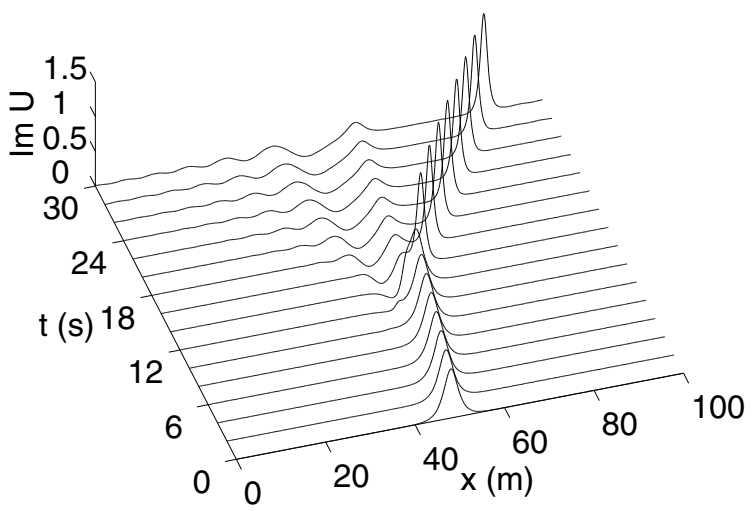

(b)

Fig. 6. Real (a) and imaginary part (b) of the numerical solution obtained for the interaction between $y$-polarized and $z$-polarized solitary waves using the energy-preserving scheme with $h=0.2, \Delta t=0.1, x_{1}=25 \mathrm{~m}, x_{2}=48 \mathrm{~m}, T=30 \mathrm{~s}$, and $0 \leq x \leq 100$. 


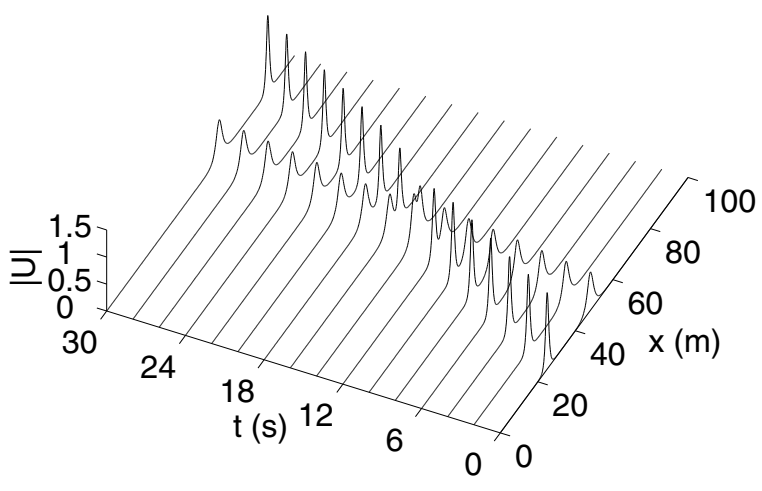

(a)

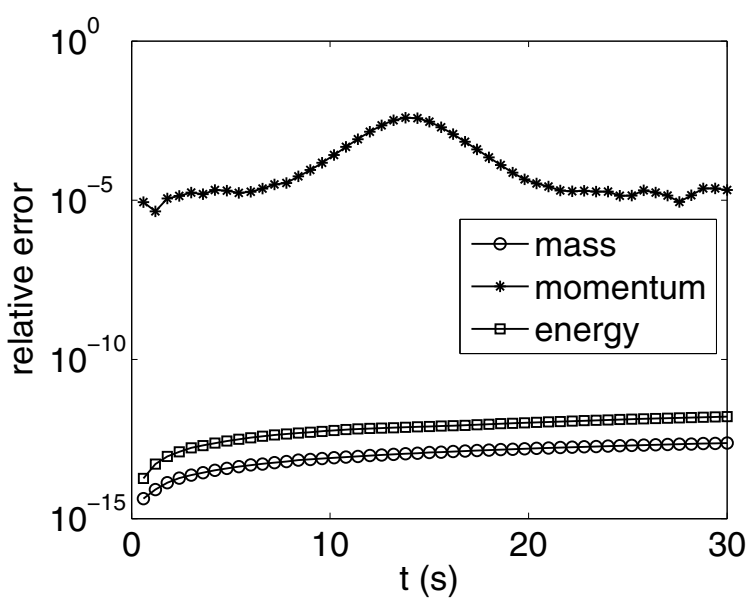

(b)

Fig. 7. Modulus (a) and relative errors of the invariants (b) of the numerical solution obtained for the interaction between two $y$-polarized solitary waves using the energy-preserving scheme with $h=0.2, \Delta t=0.1, x_{1}=25 \mathrm{~m}, x_{2}=48 \mathrm{~m}, T=30 \mathrm{~s}$, and $0 \leq x \leq 100$.

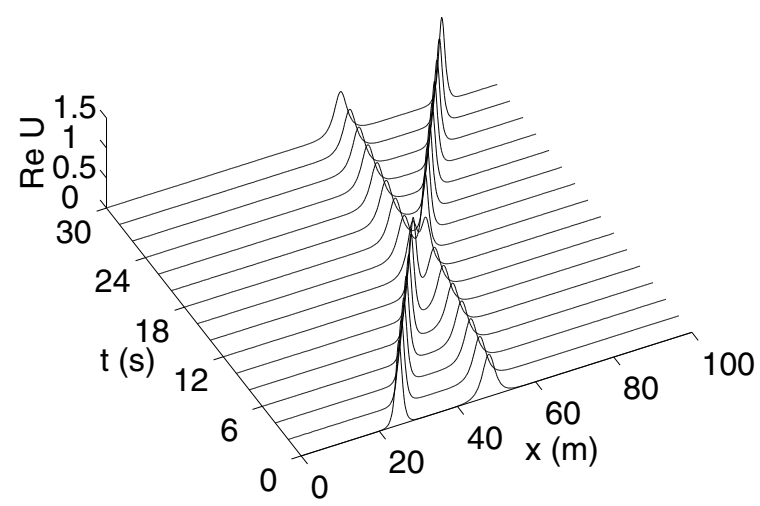

(a)

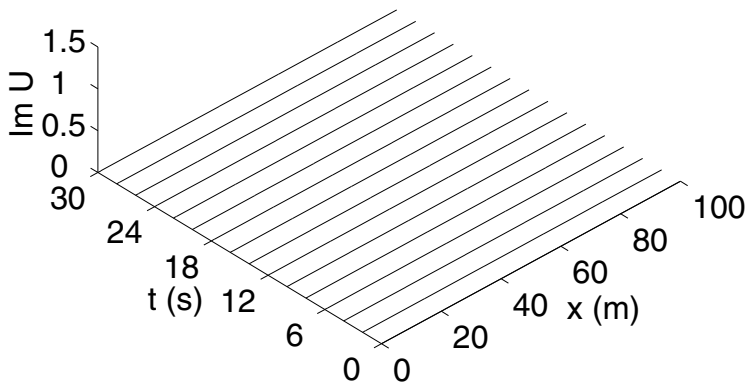

(b)

Fig. 8. Real (a) and imaginary part (b) of the numerical solution obtained for the interaction between two $y$-polarized solitary waves using the energy-preserving scheme with $h=0.2, \Delta t=0.1, x_{1}=25 \mathrm{~m}, x_{2}=48 \mathrm{~m}, T=30 \mathrm{~s}$, and $0 \leq x \leq 100$.

It is also noted that the distance between two solitary waves becomes larger and larger as time increases. On the other hand, during the whole process, the relative errors of the total mass and energy approach machine precision while the relative error of the total momentum is bounded. In addition, the real and imaginary parts of the solution exhibit the same behavior, see Fig. 10.

\section{Conclusion}

We have presented an accurate energy-preserving finite volume element scheme for the CMKDV equation. The method is based on the use of a discrete variational derivative method (DVDM) in time and a finite volume element approximation in space. The resulting energy-preserving scheme can exactly conserve the mass and energy at the discrete level. In addition, the scheme shows good long time behaviors. The energy-preserving scheme is compared with the momentum-preserving scheme and the implicit midpoint scheme for solving the CMKDV equation. The results show that the energy-preserving scheme is the best of the three proposed methods when it comes to energy conserving properties.

\section{Acknowledgment}

This work was supported in part by the PhD Start-up Fund of Wuyi University (grant no. YJ201702), the Education Foundation of Fujian Province for Young Teachers (grant no. JA14319) and the Undergraduate Technology Innovation Project of Fujian Province (grant no. SJ2011019). The authors are grateful to reviewers for their careful reading of the paper as well as their comments and suggestions. 


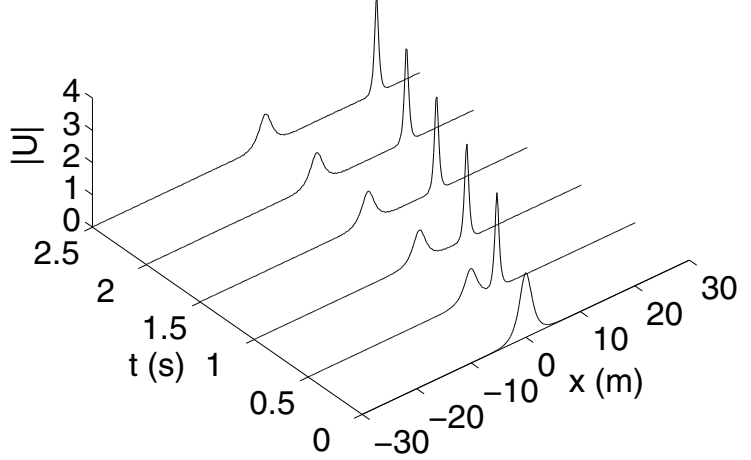

(a)

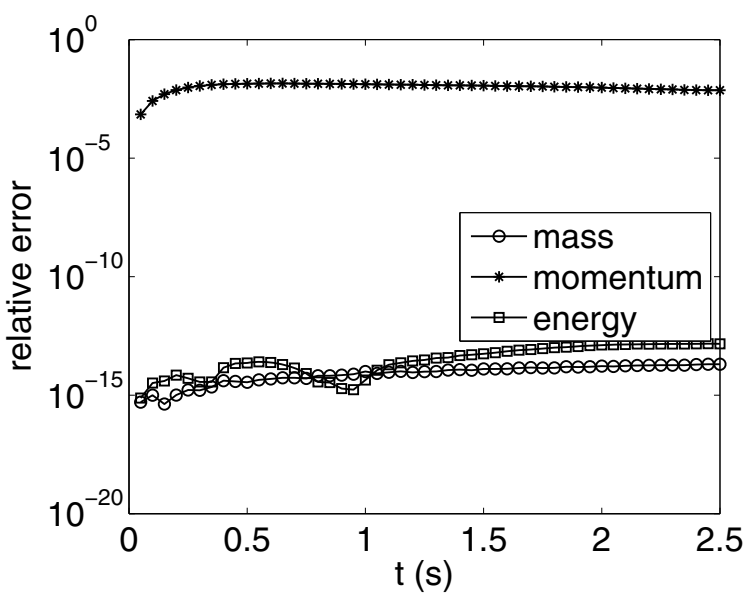

(b)

Fig. 9. Modulus (a) and relative errors of the invariants (b) of the numerical solution obtained for the wave generation simulation using the energy-preserving scheme with $h=0.2, \Delta t=0.01, T=2.5 \mathrm{~s}$, and $-30 \leq x \leq 30$.

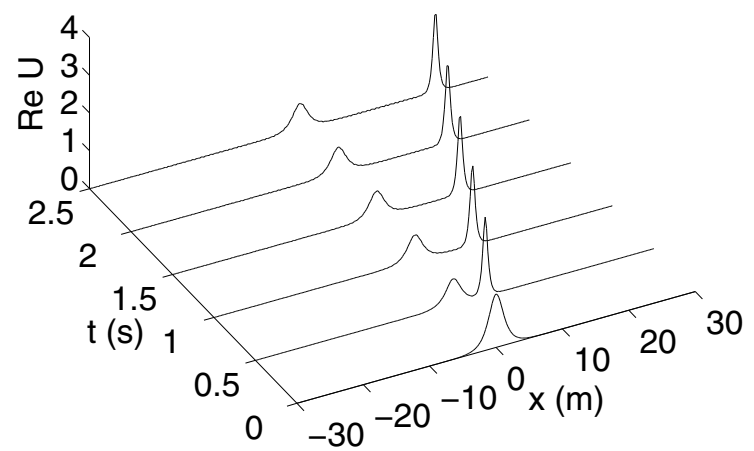

(a)

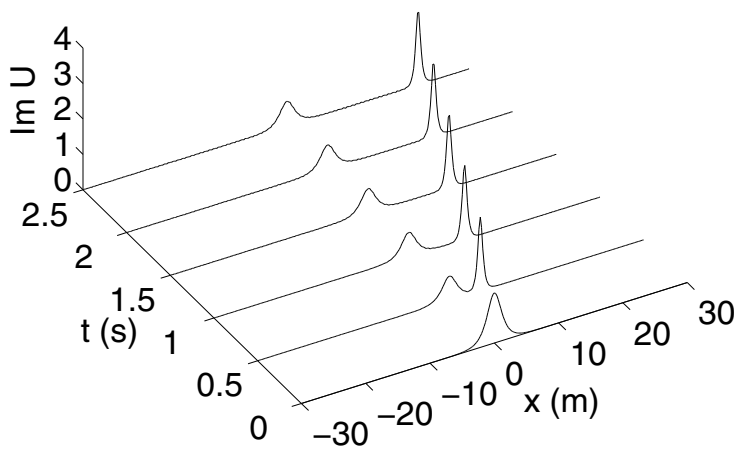

(b)

Fig. 10. Real (a) and imaginary part (b) of the numerical solution obtained for the wave generation simulation using the energypreserving scheme with $h=0.2, \Delta t=0.01, T=2.5 \mathrm{~s}$, and $-30 \leq x \leq 30$.

\section{References}

Bank, R.E. and Rose, D.J. (1987). Some error estimates for the box methods, SIAM Journal on Numerical Analysis 24(4): 777-787.

Cai, J.X. and Miao, J. (2012). New explicit multisymplectic scheme for the complex modified Korteweg-de Vries equation, Chinese Physics Letters 29(3): 030201.

Cai, Z.Q. (1991). On the finite volume element method, $\mathrm{Nu}$ merische Mathematik 58(7): 713-735.

Costa, R., Machado, G.J. and Clain, S. (2015). A sixth-order finite volume method for the 1D biharmonic operator: Application to intramedullary nail simulation, International Journal of Applied Mathematics and Computer Science 25(3): 529-537, DOI: 10.1515/amcs-2015-0039.

Erbay, H.A. (1998). Nonlinear transverse waves in a generalized elastic solid and the complex modified Korteweg-de Vries equation, Physica Scripta 58(1): 9-14.

Erbay, S. and Suhubi, E.S. (1989). Nonlinear wave propagation in micropolar media. II: Special cases, solitary waves and
Painlevé analysis, International Journal of Engineering Science 27(8): 915-919.

Ewing, R., Lin, T. and Lin, Y. (2000). On the accuracy of the finite volume element method based on piecewise linear polynomials, SIAM Journal on Numerical Analysis 39(6): 1865-1888.

Furihata, D. and Matsuo, T. (2010). Discrete Variational Derivative Method: A Structure-Preserving Numerical Method For Partial Differential Equations, CRC Press, London.

Furihata, D. and Mori, M. (1996). A stable finite difference scheme for the Cahn-Hilliard equation based on the Lyapunov functional, Zeitschrift fur Angewandte Mathematik und Mechanik 76(1): 405-406.

Gorbacheva, O.B. and Ostrovsky, L.A. (1983). Nonlinear vector waves in a mechanical model of a molecular chain, Physica D 8(1-2): 223-228.

Hackbusch, W. (1989). On first and second order box schemes, Computing 41(4): 277-296. 
Ismail, M.S. (2008). Numerical solution of complex modified Korteweg-de Vries equation by Petrov-Galerkin method, Applied Mathematics and Computation 202(2): 520-531.

Ismail, M.S. (2009). Numerical solution of complex modified Korteweg-de Vries equation by collocation method, Communications in Nonlinear Science and Numerical Simulation 14(3): 749-759.

Karney, C.F.F., Sen, A. and Chu, F.Y.F. (1979). Nonlinear evolution of lower hybrid waves, Physics of Fluids 22(5): 940-952.

Koide, S. and Furihata, D. (2009). Nonlinear and linear conservative finite difference schemes for regularized long wave equation, Japan Journal of Industrial and Applied Mathematics 26(1): 15-40.

Korkmaz, A. and Dağ, I. (2009). Solitary wave simulations of complex modified Korteweg-de Vries equation using differential quadrature method, Computer Physics Communications 180(9): 1516-1523.

Li, R.H., Chen, Z.Y. and Wu, W. (2000). Generalized Difference Methods for Differential Equations: Numerical Analysis of Finite Volume Methods, Marcel Dekker Inc., New York, NY.

Matsuo, T. and Furihata, D. (2001). Dissipative or conservative finite difference schemes for complex-valued nonlinear partial differential equations, Journal of Computational Physics 171(2): 425-447.

Matsuo, T. and Kuramae, H. (2012). An alternating discrete variational derivative method, AIP Conference Proceedings 1479: 1260-1263.

Miyatake, Y. and Matsuo, T. (2014). A general framework for finding energy dissipative/conservative $H^{1}$-Galerkin schemes and their underlying $H^{1}$-weak forms for nonlinear evolution equations, BIT Numerical Mathematics 54(4): 1119-1154.

Muslu, G.M. and Erabay, H.A. (2003). A split-step Fourier method for the complex modified Korteweg-de Vries equation, Computers \& Mathematics with Applications 45(1): 503-514.
Uddin, M., Haq, S. and Islam, S.U. (2009). Numerical solution of complex modified Korteweg-de Vries equation by mesh-free collocation method, Computers \& Mathematics with Applications 58(3): 566-578.

Wang, Q.X., Zhang, Z.Y., Zhang, X.H. and Zhu, Q.Y. (2014). Energy-preserving finite volume element method for the improved Boussinesq equation, Journal of Computational Physics 270: 58-69.

Yaguchi, T., Matsuo, T. and Sugihara, M. (2010). An extension of the discrete variational method to nonuniform grids, Journal of Computational Physics 229(11): 4382-4423.

Yan, J.L., Zhang, Q., Zhu, L. and Zhang, Z.Y. (2016). Two-grid methods for finite volume element approximations of nonlinear Sobolev equations, Numerical Functional Analysis and Optimization 37(3): 391-414.

Zhang, Z.Y. and Lu, F.Q. (2012). Quadratic finite volume element method for the improved Boussinesq equation, Journal of Mathematical Physics 53(1): 013505.

Jinliang Yan received his $\mathrm{PhD}$ and Master's degrees in computational mathematics at Nanjing Normal University, China. He also holds a teaching position in the Department of Mathematics and Computing at Wuyi University. His research interests include structure-preserving algorithms, numerical solutions of partial differential equations and computing sciences. He is also the author of numerous articles, mainly in the field of energy-preserving methods and their applications.

Lianghong Zheng holds an MSc degree in computer science and technology from Minnan Normal University, China. Also, she is a teacher in the Department of Information and Technology at Nanping No. 1 Middle School in Fujian, China. Her research interests include artificial intelligence, robot competition and video production.

Received: 22 October 2016 Revised: 25 February 2017 Re-revised: 6 April 2017 Accepted: 10 April 2017 